\title{
ON GENERALIZING THE NIELSEN COINCIDENCE THEORY TO NON-ORIENTED MANIFOLDS
}

\author{
JERZY JEZIERSKI \\ Department of Mathematics, Agriculture University \\ Nowoursynowska 166, 02-766 Warszawa, Poland \\ E-mail: jezierski@alpha.sggw.waw.pl
}

\begin{abstract}
We give an outline of the Nielsen coincidence theory emphasizing differences between the oriented and non-oriented cases.

1. Introduction. The coincidence theory considers the following problem. Given a pair of continuous maps between topological spaces $f, g: M \rightarrow N$. Describe the coincidence set $C(f, g)=\{x \in M ; f x=g x\}$ of this pair. More precisely we are interested in those properties of $C(f, g)$ which do not change after homotopies of $f$ and $g$.

This theory is a common generalization of two classical theories:

Fixed points $(M=N$ and $g=$ identity map). Then $C(f, g)=F i x(f)=\{x \in M$; $f x=x\})$ is fixed point set.

Roots ( $g$ constant $\left.g x \equiv y_{0} \in N\right)$. Then $C(f, g)=f^{-1}\left(y_{0}\right)$.

We will give here the definition and some basic properties of the Nielsen coincidence number $N(f, g)$. It is a non-negative integer which is a homotopy invariant and a lower bound of the cardinality of the coincidence set: $N(f, g) \leq \# C(f, g)$. Our main idea is to describe so called "defective classes" and exhibit them as the obstruction to generalizing coincidence theorems to the non-orientable manifolds. This paper is not self-contained: to make it short we often only sketch proofs omitting longer (usually technical) parts. For details we send to the referenced papers. Our main idea is to inform briefly the reader familiar with fixed point theory about generalizations of this theory to coincidences.

In sections 2, 3 we recall the definition of the Nielsen number in the oriented and in the non-oriented case respectively. Then in section 4 we introduce defective classes. In section 5 we consider the non-oriented manifolds but we make some assumptions on one of the maps. This assumption allows us to extend the Lefschetz coincidence formula
\end{abstract}

1991 Mathematics Subject Classification: Primary 55M20.

The paper is in final form and no version of it will be published elsewhere. 
beyond the oriented case (section 6). In the last two sections we recall two effective methods of computation of the Nielsen number: fibre maps and covering spaces. We sketch fragments of these computations illustrating problems arising when we drop the orientability assumption.

The first application of algebraic topology to coincidences was probably that by Solomon Lefschetz in 1926 [L]. For a pair of maps $f, g: M \rightarrow N$ between oriented closed manifolds of the same dimension he defined an integer number. This number, called the Lefschetz number today, is denoted $L(f, g)$ and satisfies

Theorem 1.1 (Lefschetz Coincidence Theorem). If $f, g: M \rightarrow N$ between oriented manifolds of the same dimension and $L(f, g) \neq 0$ then any pair of maps $f^{\prime}, g^{\prime}$ homotopic to $f, g$ has a coincidence point.

(Here the pairs are homotopic if $f$ is homotopic to $f^{\prime}$ and $g$ is homotopic to $g^{\prime}$ ). Since that time this theorem has been widely applied, mainly for fixed points $(g=i d)$. However we would like to remind the reader that in the original form this theorem was formulated for coincidences.

At the same time Jacob Nielsen introduced in [N1], [N2] a theory estimating the number of fixed points. The main result of those papers may be formulated as follows. For any self-map of a finite polyhedron $f: M \rightarrow M$ a non-negative integer $N(f)$ is defined (Nielsen number). This number satisfies three conditions:

- $N(f)$ is a homotopy invariant,

- $N(f)$ is a lower bound of the number of fixed points: $N(f) \leq \#$ Fix $(f)$,

- $N(f)$ is computable in some situations.

Thus $N(f)$ informs about the minimal number of fixed points in the homotopy class of $f$. It is natural to ask about a similar lower bound of the number of coincidences of a pair $f, g: M \rightarrow N$.

2. Nielsen number in the oriented case. Such a generalization requires serious restrictions on the spaces $M$ and $N$. First we show how to define the Nielsen coincidence number for pairs of maps $f, g: M \rightarrow N$ where $M, N$ are closed oriented manifolds of the same dimension. This was done by Helga Schirmer in her Ph.D. thesis in 1955 [Sch].

We start by defining the coincidence index. Let $U \subset M$ be an open subset for which $U \cap C(f, g)$ is compact. Consider a sequence of homomorphisms of homology groups (with integer coefficients):

$$
\begin{aligned}
H_{n} M \rightarrow H_{n}(M, M-(U \cap C(f, g))) \stackrel{\text { exc }}{=} H_{n}(U, U-(U \cap C(f, g))) \stackrel{(f, g)_{*}}{\longrightarrow} \\
H_{n}(N \times N, N \times N-\Delta N) \rightarrow \mathbf{Z}
\end{aligned}
$$

where the first homomorphism is induced by the inclusion of pairs $(M, \emptyset) \subset(M, M-(U \cap$ $C(f, g))$ ), the second is the excision isomorphism, the third is induced by the map $U \ni$ $x \rightarrow(f x, g x) \in N \times N$ and the last is the evaluation of the Thom class $U \in H^{n}(N \times N)$ corresponding to the given orientation of $N$. We define the coincidence index as the image of the fundamental class $z_{M} \in H_{n}(M, \mathbf{Z})$ under this sequence of homomorphisms. We write $\operatorname{ind}(f, g ; U)$. Notice that $i n d(f, g ; U) \neq 0$ implies a coincidence point in $U$. 
This definition has the following geometric interpretation. We recall that if $V$ is an oriented closed $n$-manifold, $z_{V} \in H_{n}(V)$ denotes its fundamental class and $i: V \rightarrow N \times N$ is a map transverse to the diagonal $\Delta N$ then the evaluation of the Thom class on the homology class $i_{*}\left(z_{V}\right) \in H_{n}(N \times N)$ is the algebraic sum of intersection points of the map $i$ with $\Delta N$. In the above sequence defining the coincidence index (for $U=M$ ) we obtain the evaluation of the Thom class of $N$ at $(f, g)_{*}\left(z_{M}\right)$ where the map $(f, g): M \rightarrow N \times N$ is given by $(f, g)(x)=(f(x), g(x))$. Thus the coincidence index may be interpreted as the algebraic number of intersections of the map $(f, g)$ with the diagonal $\Delta N$.

Now we define an equivalence relation on the coincidence set $C(f, g)$ (Nielsen relation). Two points $x, y \in C(f, g)$ are equivalent if there exists a path $\omega$ joining them (i.e. $\omega(0)=$ $x, \omega(1)=y$ ) such that the paths $f \omega$ and $g \omega$ are fixed end point homotopic. This relation divides the coincidence set into equivalence classes (Nielsen classes). It is easy to see that each Nielsen class is a closed-open subset of $C(f, g)$. In particular their number is finite since $C(f, g)$ is compact.

The Nielsen classes can be also interpreted as follows. Let us fix universal coverings $p_{M}: \tilde{M} \rightarrow M$ and $p_{N}: \tilde{N} \rightarrow N$. As in the case of fixed points one may check that a subset $A \subset C(f, g)$ is a Nielsen class if and only if there exist lifts $\tilde{f}, \tilde{g}: \tilde{M} \rightarrow \tilde{N}$ of $f, g$ respectively satisfying $A=p_{M}(C(\tilde{f}, \tilde{g})$.

For any closed-open subset $A \subset C(f, g)$ we take an open set $U$ satisfying $U \cap C(f, g)=$ $A$ and we define the index of this set as $\operatorname{ind}(f, g ; U)$. We write $\operatorname{ind}(f, g ; A)=\operatorname{ind}(f, g ; U)$. It is easy to check that this definition does not depend on the choice of $U$. We call a Nielsen class essential if its index is not zero and we define the Nielsen number $N(f, g)$ of the pair $f, g$ as the number of essential classes. Clearly

$$
N(f, g) \leq \# C(f, g) .
$$

On the other hand $N(f, g)$ is a homotopy invariant:

LEMMA 2.1. If $f_{0}, g_{0}$ and $f_{1}, g_{1}$ are homotopic pairs of maps between oriented closed manifold then $N\left(f_{0}, g_{0}\right)=N\left(f_{1}, g_{1}\right)$.

SkEtch OF PROOF. Let $f_{t}, g_{t}$ be a required pair of homotopies. Let $A_{0}$ be a Nielsen class of $f_{0}, g_{0}$. For any $t \in[0,1]$ we define

$A_{t}=\left\{x \in M\right.$; there exists a path $\omega: I \rightarrow M$ such that $\omega(0) \in A_{0}, \omega(1)=x$ and the paths $s \rightarrow f_{s t}(\omega(s))$ and $s \rightarrow g_{s t}(\omega(s))$ are homotopic in $\left.N\right\}$. Notice that

- $A_{t}$ is a Nielsen class of $f_{t}, g_{t}$ or is empty.

- $A=\bigcup_{t \in I} A_{t}$ is a clopen subset of $\bigcup_{t \in I} C\left(f_{t}, g_{t}\right)$.

- For any $t_{0} \in I$ there exist an open set $U \subset M$ and $\epsilon>0$ such that $U \cap C\left(t_{t}, g_{t}\right)=A_{t}$ for any $t \in\left(t_{0}-\epsilon, t_{0}+\epsilon\right)$.

Now we may apply the neighbourhood $U$ in the sequence defining $i n d\left(f_{t}, g_{t} ; A_{t}\right)$ and we get that all these indices are equal for $t \in\left(t_{0}-\epsilon, t_{0}+\epsilon\right)$. Thus the function

$$
[0,1] \ni t \rightarrow \operatorname{ind}\left(f_{t}, g_{t} ; A_{t}\right) \in \mathbf{Z}
$$

is locally constant on $[0,1]$ hence it is constant and $\operatorname{ind}\left(f_{0}, g_{0} ; A_{0}\right)=\operatorname{ind}\left(f_{1}, g_{1} ; A_{1}\right)$.

Now we see that the correspondence $A_{0} \leftrightarrow A_{1}$ defines a bijection between essential Nielsen classes of pairs $f_{0}, g_{0}$ and $f_{1}, g_{1}$, which implies $N\left(f_{0}, g_{0}\right)=N\left(f_{1}, g_{1}\right)$. 
The next question is whether the above Nielsen number is the best lower bound of the number of coincidences in a homotopy class. H. Schirmer proved [Sch] the following generalization of the Wecken theorem:

THEOREM 2.2. Let $M, N$ be closed oriented topological manifolds of dimension $n \geq 3$. Then for any pair of maps $f, g: M \rightarrow N$ there exists a pair $f^{\prime}, g^{\prime}$ which has exactly $N(f, g)$ coincidence points.

In the proof given in [Sch] the maps $f, g$ are replaced by a transverse pair and then the Whitney trick is used to reduce the number of coincidence points to the minimal one.

3. Nielsen number on non-oriented manifolds. Let us drop the orientability assumption on $M$ and $N$. Then the Nielsen relation divides $C(f, g)$ into classes and it remains to define essential classes. In section 5 we show that one may extend the coincidence index to pairs $f, g$ satisfying some additional conditions. However the following example suggests that such an extension is not possible in general (without losing homotopy and localization properties [Dl]).

EXAMPLE 3.1. Consider the projective plane $R P^{2}=D^{2} / \sim$ as the quotient space of the 2-disk $D^{2}=\{z \in C ;|z|=1\}$ where $z \sim z^{\prime}$ iff $|z|=\left|z^{\prime}\right|=1$ and $z^{\prime}=-z$. Define maps $f, g: R P^{2} \rightarrow R P^{2}$ by $f[z]=[0], g[z]=\left[z^{2}\right]$. Then $C(f, g)=\{[0]\}$ and the restriction of these maps to a neighbourhood of [0] has index \pm 2 (the sign depends on the chosen local orientations). On the other hand the homotopy $g_{t}[z]=\left[t z^{2}\right]$ contracts $g$ to a constant map. Thus $f, g$ is homotopic to a coincidence free pair.

However there exists a coincidence semi-index which allows defining essential classes in the non-oriented case. The definition of this semi-index, inspired by [Ji2], is given in [DJ] and [Je4]. We will here sketch the construction of the semi-index for smooth manifolds as in [DJ]. This construction can be reformulated for topological manifolds by using microbundles instead of tangent bundles [Je4].

Consider a pair of maps $f, g: M \rightarrow N$ between closed smooth manifolds. This pair will be called transverse if the maps $f, g$ are smooth in a neighbourhood of $C(f, g)$ and the map $M \ni x \rightarrow(f x, g x) \in N \times N$ is transverse to the diagonal $\Delta N \subset N \times N[\mathrm{H}]$. One may check that the pair $f, g$ is transverse iff for any coincidence point $x \in C(f, g)$ the difference of the tangent maps $T_{x} f-T_{x} g: T_{x} M \rightarrow T_{f x} N$ is an epimorphism. By general theorems any pair is homotopic to a transverse pair $[\mathrm{H}]$.

Let $f, g: M \rightarrow N$ be a transverse pair of maps between closed smooth $n$-manifolds. Then any coincidence point is isolated in $C(f, g)$. Let $x_{0}, x_{1} \in C(f, g)$ and let $\omega$ be a path establishing the Nielsen relation between these points. Let $\alpha_{0}$ denote an orientation of the vector space $T_{x_{0}} M$ and let $\alpha_{1}$ be the orientation of $T_{x_{1}}$ obtained under the shift of $\alpha_{0}$ along $\omega$. Let $\beta_{0}$ denote the orientation of $T_{f x_{0}} N$ obtained as the image of $\alpha_{0}$ by the isomorphism $T_{f x_{0}}-T_{g x_{0}}$. Let $\beta_{1}$ be the shift of $\beta_{0}$ along $f \omega$ (or equivalently $g \omega$ ). We will say that $\omega$ establishes the $R$-relation between the points $x_{0}$ and $x_{1}$ if the image of $\alpha_{1}$ under the isomorphism $T_{f x_{1}}-T_{g x_{1}}$ is opposite to the orientation $\beta_{1}$. We call two coincidence points $x, y$ (from the same Nielsen class) R-related if there exists a path establishing the 
R-relation between them. We will then write $x R y$. Notice that the R-relation is not an equivalence relation. In fact the relation opposite to it is an equivalence relation.

Consider a subset $A \subset C(f, g)$ of a transverse pair $f, g$. Let us present its elements as

$$
A=\left\{x_{1}, y_{1}, \ldots, x_{k}, y_{k} ; z_{1}, \ldots z_{s}\right\}
$$

where $x_{i} R y_{i}$ for $i=1, \ldots, k$ but $z_{i} R z_{j}$ for no $i \neq j$. The elements $z_{1}, \ldots, z_{s}$ are called free in this presentation. One may check that the number of free elements is the same for all presentations of $A[\mathrm{DJ}]$. Now we may define the semi-index of $A$ as the number of free points: $\mid$ ind $\mid(f, g ; A)=s$.

Lemma 3.2. Let $F, G: M \times I \rightarrow N$ be a pair of transverse homotopies and let Nielsen classes $A_{0}, A_{1}$ of pairs $f_{0}, g_{0}$ and $f_{1}, f_{1}$ correspond to each other under this homotopy. Then

$$
\mid \text { ind }\left|\left(f_{0}, g_{0} ; A_{0}\right)=\right| \text { ind } \mid\left(f_{1}, g_{1} ; A_{1}\right) .
$$

Sketch of PROOF. By the transversality $C(F, G)$ is a one-dimensional submanifold in $M \times I$.

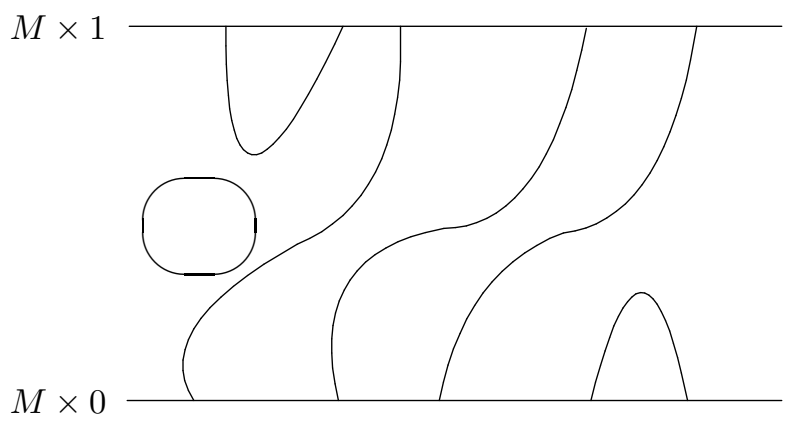

It turns out that:

- If both ends of a component $\omega \subset C(f, g)$ are contained in $M \times 0$ (or $M \times 1)$ then these ends are R-related as coincidence points of $f_{0}, g_{0}$ (or $f_{1}, g_{1}$ ) (the R-relation is determined by the path $p \omega$ where $p: M \times I \rightarrow M$ denotes the natural projection).

- If $\omega, \omega^{\prime} \subset C(F, G)$ are components with ends $\partial \omega=\left\{\left(x_{0}, 0\right),\left(x_{1}, 1\right)\right\}, \partial \omega^{\prime}=$ $\left\{\left(y_{0}, 0\right),\left(y_{1}, 1\right)\right\}$ then $x_{0} R y_{0}$ implies $x_{1} R y_{1}$ (if $\alpha$ establishes $x_{0} R y_{0}$ then $(p \omega)^{-1} * \alpha *$ $\left(p \omega^{\prime}\right)$ establishes $\left.x_{1} R y_{1}\right)$.

These two properties give rise to presentations of $A_{0}$ and $A_{1}$ with the same number of fixed points.

Thus the essential classes of $f_{0}, g_{0}$ are in a bijection with the essential classes of $f_{1}, g_{1}$.

Corollary 3.3. Let $F, G: M \times I \rightarrow N$ be a pair of transverse homotopies between pairs $f_{0}, g_{0}$ and $f_{1}, g_{1}$. Then

$$
N\left(f_{0}, g_{0}\right)=N\left(f_{1}, g_{1}\right) .
$$

This corollary allows us to extend the definition of the coincidence semi-index to an arbitrary continuous pair $f, g$. Let $A \subset C(f, g)$ be a Nielsen class. Let $f^{\prime}, g^{\prime}$ be a 
transverse approximation of $f, g$ and let $A^{\prime} \subset C\left(f^{\prime}, g^{\prime}\right)$ be the corresponding class. We define

$$
\mid \text { ind }|(f, g ; A)=| \text { ind } \mid\left(f^{\prime}, g^{\prime} ; A^{\prime}\right) .
$$

If $f^{\prime \prime}, g^{\prime \prime}$ is another transverse approximation and $A^{\prime \prime}$ is the corresponding Nielsen class then there is a transverse homotopy from $f^{\prime}, g^{\prime}$ to $f^{\prime \prime}, g^{\prime \prime}$ sending $A^{\prime}$ to $A^{\prime \prime}$. By the above corollary $\mid$ ind $\left|\left(f^{\prime}, g^{\prime} ; A^{\prime}\right)=\right| i n d \mid\left(f^{\prime \prime}, g^{\prime \prime} ; A^{\prime \prime}\right)$ hence the extension of the definition is correct.

If the manifolds $M$ and $N$ are oriented and the maps $f, g$ are transverse then $\operatorname{ind}(f, g ; x)= \pm 1$ at any coincidence point $x$. Moreover analysis of the R-relation shows that for two points $x_{0}, x_{1} \in C(f, g)$ from the same Nielsen class, $x_{0} R x_{1}$ iff $\operatorname{ind}\left(f, g ; x_{0}\right)=$ $-\operatorname{ind}\left(f, g ; x_{1}\right)$. Thus we obtain

Corollary 3.4. In the oriented case the semi-index of any Nielsen class A equals the absolute value of the coincidence index:

$$
|\operatorname{ind}|(f, g ; A)=|\operatorname{ind}(f, g ; A)| .
$$

Consider again the general (non-oriented) situation. We call a Nielsen class essential if its semi-index is not zero and we define the Nielsen number $N(f, g)$ of the pair $f, g$ as the number of essential classes. By the above corollary the definition is consistent with the oriented case.

THEOREM 3.5. Any pair of maps $f, g: M \rightarrow N$ between closed smooth manifolds has at least $N(f, g)$ coincidence points.

Proof. This is evident when the pair $f, g$ is transverse. In the general case assume that $\# C(f, g)=k<N(f, g)$. For any $x \in C(f, g)$ we choose a contractible neighbourhood $W_{x}$ of $f x=g x \in N$. Then we take a neighbourhood $V_{x}$ of $x \in M$ so small that $f\left(c l V_{x}\right) \cup$ $g\left(c l V_{x}\right) \subset W_{x}$. Let $f_{t}, g_{t}$ be a compact homotopy supported in $\bigcup\left\{V_{x} ; x \in C(f, g)\right\}$ from $f, g$ to a transverse pair. Since this homotopy may be arbitrarily small, we may assume that $f_{t}\left(c l V_{x}\right) \cup g_{t}\left(c l V_{x}\right) \subset W_{x}$ hence $C(f, g) \cap V_{x}$ belongs to one Nielsen class. Now the transverse pair $f_{1}, g_{1}$ contains at most $k$ non-empty classes contradicting $N\left(f_{1}, g_{1}\right)=$ $N(f, g)>k$.

REMARK 3.6. The coincidence semi-index does not have the additivity property: if for example $x, y$ are R-related then

$$
\mid \text { ind }|(f, g:\{x, y\})=0<1+1=| \text { ind }|(f, g:\{x\})+| \text { ind } \mid(f, g:\{y\}) .
$$

One the other hand as in the oriented case the Nielsen number is the best lower bound in dimensions greater than or equal to three.

Theorem 3.7. Let $M, N$ be closed smooth manifolds of dimension $n \geq 3$. Then for any pair of maps $f, g: M \rightarrow N$ there exists a pair $f^{\prime}, g^{\prime}$ which has exactly $N(f, g)$ coincidence points.

The proof follows that in the oriented case. We use a local version of the Whitney lemma [DJ]. This theorem also holds for topological manifolds [Je4]. 
4. Self-reducing coincidence points. Consider the following question. Can a coincidence point $x$ reduce itself: $x R x$ ? This means the existence of a loop $\alpha$ based at $x$ such that $f \alpha \sim g \alpha$ and exactly one of the loops $\alpha$ or $f \alpha$ preserves orientation. Such an $x$ will be called a self-reducing coincidence point.

ExAmple 4.1. Let $f, g: M \rightarrow S^{n}$ where $M$ is a non-orientable $n$-manifold $(n \geq 2)$. Let $\alpha$ be a loop reversing orientation of $M$ and based at $x_{0} \in C(f, g)$. Since $S^{n}$ is simply-connected, $f \alpha$ and $g \alpha$ are contractible, which gives $x_{0} R x_{0}$.

LEMmA 4.2. If a Nielsen class $A$ contains a self-reducing point $x_{0}$ then any two points in $A$ are $R$-related.

Proof. Let a path $\alpha$ establish $x_{0} R x_{0}$ and let $\beta$ establish the Nielsen relation between $x_{0}$ and another point $x_{1} \in A$. If $\beta$ does not establish $x_{0} R x_{1}$ then $\alpha * \beta$ does. Thus $x_{0} R x_{1}$ for any $x_{1} \in A$. On the other hand if $\alpha_{i}$ establishes $x_{0} R x_{i}(i=1,2)$ then $\alpha_{1}^{-1} * \alpha_{0} * \alpha_{2}$ establishes $x_{1} R x_{2}$.

Thus if a Nielsen class $A$ of a transverse pair $f, g$ contains a self-reducing point then

$$
|i n d|(f, g ; A)= \begin{cases}0 & \text { if } \# A \text { is even } \\ 1 & \text { if } \# A \text { is odd }\end{cases}
$$

The self-reducing property can also be expressed by the fundamental groups. We denote by $\pi_{1}^{+}\left(M ; x_{0}\right)$ the subgroup of elements preserving orientation and put $C\left(f_{\#}, g_{\#}\right)_{x_{0}}$ $\left.=\left\{\alpha \in \pi_{1}\left(M ; x_{0}\right) ; f \alpha=g \alpha\right)\right\}$ (for $x_{0} \in C(f, g)$ ). Then it is easy to check that

$$
x_{0} R x_{0} \text { iff } f_{\#}^{-1}\left(\pi_{1}^{+}\left(N ; f x_{0}\right)\right) \cap C\left(f_{\#}, g_{\#}\right)_{x_{0}} \neq C\left(f_{\#}, g_{\#}\right)_{x_{0}} \cap \pi_{1}^{+}\left(M ; x_{0}\right) .
$$

Definition 4.3. A Nielsen class $A$ of a pair of continuous maps is called defective if for some (hence any) point $x_{0} \in A, x_{0} R x_{0}$.

One may check that defectiveness is a homotopy invariant property. Obviously in the orientable case no Nielsen class is defective. In the next sections we will show that defective classes make the non-oriented case essentially different from the oriented case.

5. Orientation true maps. Consider the sequence defining the coincidence index (section 2). If $M$ is non-orientable then $H_{n}(M ; \mathbf{Z})=\mathbf{Z}_{2}$ hence the homomorphism can be non-zero only when we use $\mathbf{Z}_{2}$ coefficients. Then we obtain

$$
\begin{array}{r}
H_{n}\left(M ; \mathbf{Z}_{2}\right) \rightarrow H_{n}\left(M, M-(U \cap C(f, g)) ; \mathbf{Z}_{2}\right) \stackrel{e x c}{=} H_{n}\left(U, U-(U \cap C(f, g)) ; \mathbf{Z}_{2}\right) \stackrel{(f, g)_{*}}{\longrightarrow} \\
H_{n}\left(N \times N, N \times N-\Delta N ; \mathbf{Z}_{2}\right) \rightarrow \mathbf{Z}_{2}
\end{array}
$$

where the last homomorphism is the evaluation of the $\mathbf{Z}_{2}$-Thom class $U_{2} \in H^{n}(N \times$ $N ; \mathbf{Z}_{2}$ ). The image of the unique non-zero element $z_{M} \in H_{n}\left(M ; \mathbf{Z}_{2}\right)$ (Thom class) is called the index modulo 2. This index is defined for all manifolds and is a homotopy invariant satisfying the additivity property. We will denote it by $\operatorname{ind}_{2}(f, g ; U)$. Now it is easy to observe that if the maps $f, g$ are transverse in $U$ then

$$
\text { ind }_{2}(f, g ; U)= \begin{cases}0 & \text { if } \# C(f, g) \text { is even, } \\ 1 & \text { if } \# C(f, g) \text { is odd. }\end{cases}
$$


It is also easy to observe that in the oriented case the unique epimorphism $\mathbf{Z} \rightarrow \mathbf{Z}_{2}$ sends ind $(f, g ; U)$ to $i_{n} d_{2}(f, g ; U)$. Unfortunately this index loses all "even" information about $C(f, g)$.

EXAMPLE 5.1. Let $p: S^{2 n} \rightarrow R P^{2 n}$ denote the natural covering map. Since $S^{2 n} \subset$ $R^{2 n+1}$, we will denote its points by $\left(z_{1}, \ldots, z_{n}, t\right) \in C^{n} \times R$. Consider the pair $p, p$ : $R P^{2 n} \rightarrow S^{2 n}$. This pair has one non-empty Nielsen class. After a homotopy

$$
S^{2 n} \ni\left(z_{1}, \ldots, z_{n}, t\right) \stackrel{p_{t}}{\rightarrow}\left[e^{\pi i t} z_{1}, \ldots, e^{\pi i t} z_{n}, t\right] \in R P^{2 n}
$$

the coincidence set is reduced to the two points $(0, \ldots, 0,1),(0 \ldots .,-1) \in S^{2 n}$ and the pair $p, p_{t}$ is transverse. Thus $i n d_{2}(p, p)=0$. On the other hand one may check that the above two coincidence points do not reduce, which implies that $\mid$ ind $\mid(p, p)=2$. (The last may also be proved for $n \geq 2$ as follows. Suppose that these two points are R-related. Then $N(f, g)=0$ and by the Wecken theorem $(2 n \geq 3)$ there is a homotopy of the pair $p, p$ to a coincidence free pair. This homotopy lifts to a homotopy of $i d, i d$ (of $S^{2 n}$ ) to a coincidence free pair. But the last is impossible since $L(i d, i d)=\chi\left(S^{2 n}\right)=2 \neq 0$.)

Nevertheless there exists an extension of the coincidence index beyond the nonoriented case. This extension however requires some restrictions on the maps. We will have to assume that one of the maps considered, say $g$, is orientation true, i.e. for any loop $\alpha$ in $M, \alpha$ preserves orientation iff $g \alpha$ does. Notice that then no class of $f, g(f$ arbitrary) is defective. Notice that $g$ is orientation true iff $g^{*}\left(w_{i}(N)\right)=w_{1}(M)$ where $w_{1}$ stands for the the first Stiefel-Whitney class. Consider the local system of abelian groups $\Gamma_{M}$ on $M: \Gamma_{M}(x)=H_{n}(M, M-x ; \mathbf{Z})$ and the action of $\pi_{1} M$ on $\Gamma_{M}(x)$ is given by

$$
\alpha \circ \theta= \begin{cases}\theta & \text { if } \alpha \text { preserves orientation, } \\ -\theta & \text { if } \alpha \text { reverses orientation. }\end{cases}
$$

Then $H_{n}\left(M ; \Gamma_{M}\right)=\mathbf{Z}$ and we may fix a generator $z_{M} \in H_{n}\left(M ; \Gamma_{M}\right)$ (fundamental class). We notice that if a map $g: M \rightarrow N$ is orientation true then $g$ induces a homomorphism $g_{*}: H_{n}\left(M ; \Gamma_{M}\right) \rightarrow H_{n}\left(N: \Gamma_{N}\right)[\mathrm{Sp} 1]$.

For a pair $f, g$, with $g$ orientation true, we consider the sequence of homomorphisms

$$
\begin{array}{r}
H_{n}\left(M ; \Gamma_{M}\right) \rightarrow H_{n}\left(M, M-(U \cap C(f, g)) ; \Gamma_{M}\right) \stackrel{\text { exc }}{=} H_{n}\left(U, U-(U \cap C(f, g)) ; \Gamma_{M}\right) \\
\stackrel{(f, g)_{*}}{\longrightarrow} H_{n}\left(N \times N, N \times N-\Delta N ; \mathbf{Z} \times \Gamma_{N}\right) \rightarrow \mathbf{Z}
\end{array}
$$

and we define the coincidence index of the pair $f, g$ as the image of the fundamental class $z_{M}$ under the above sequence (the induced homomorphism $(f, g)_{*}$ is defined since $g$ is orientation true). In the oriented case the local systems are constant hence we obtain an extension of the ordinary index from section 2. The analogy between these two indices extends to the Lefschetz numbers and is described in the next section.

6. Lefschetz formula. The Lefschetz formula establishing equality between the index and an alternating sum of traces is the fundamental tool in effective computations in Nielsen theory. We will formulate this formula for coincidences in the oriented case after [V] and then we will generalize this formula to the pairs $f, g$ where $g$ is orientation true.

Suppose that $f, g: M \rightarrow N$ are maps between closed oriented $n$-manifolds. For any $k=0, \ldots, n$ we define a sequence of homomorphisms of (co-) homology groups with 
rational coefficients:

$$
H^{k}(N ; \mathbf{Q}) \stackrel{f^{*}}{\longrightarrow} H^{n}(M ; \mathbf{Q}) \stackrel{D_{M}}{\longrightarrow} H_{n-k}(M ; \mathbf{Q}) \stackrel{g_{*}}{\longrightarrow} H_{n-k}(N ; \mathbf{Q}) \stackrel{D_{N}^{-1}}{\longrightarrow} H^{k}(N ; \mathbf{Q})
$$

where $D_{M}, D_{N}$ denote Poincaré isomorphisms. Denote this composition by

$$
\theta_{k}=g_{*} D_{M}^{-1} f^{*} D_{N} .
$$

Let $\operatorname{tr} \theta_{k}$ denote the trace of the self-homomorphism $\theta$ of the finite dimensional $\mathbf{Q}$-space $H^{k}(N ; \mathbf{Q})$. We define the Lefschetz number of the pair $f, g$ as

$$
L(f, g)=\sum_{k=0}^{n}(-1)^{k} \operatorname{tr} \theta_{k} .
$$

TheOrem 6.1 (Lefschetz coincidence formula). For any pair of maps between closed oriented manifolds $f, g: M \rightarrow N$,

$$
L(f, g)=\operatorname{ind}(f, g) .
$$

In the non-oriented case the homomorphisms $\theta_{k}$ cannot be defined since there is no Poincaré isomorphism $H_{k}(M ; \mathbf{Q})=H^{n-k}(M ; \mathbf{Q})$ in general. Nevertheless there is a natural isomorphism (Poincaré duality) [Sp2], [GJ]

$$
D_{M}: H^{k}(M ; \mathbf{Q}) \rightarrow H_{n-k}\left(M ; \Gamma_{M} \otimes \mathbf{Q}\right) .
$$

Now we assume that $g$ is orientation true and we may define a sequence of homomorphisms

$H^{k}(N ; \mathbf{Q}) \stackrel{f^{*}}{\longrightarrow} H^{n}(M ; \mathbf{Q}) \stackrel{D_{M}}{\longrightarrow} H_{n-k}\left(M ; \Gamma_{M} \otimes \mathbf{Q}\right) \stackrel{g_{*}}{\longrightarrow} H_{n-k}\left(N ; \Gamma_{N} \otimes \mathbf{Q}\right) \stackrel{D_{N}^{-1}}{\longrightarrow} H^{k}(N ; \mathbf{Q})$

As in the oriented case we denote $\theta_{k}=g_{*} D_{M}^{-1} f^{*} D_{N}$ and we define the Lefschetz number $L(f, g)=\sum_{k=0}^{n}(-1)^{k} \operatorname{tr} \theta_{k}$.

Now section 6 from [V] can be reformulated [GJ] to give the proof of

THEOREM 6.2 (Lefschetz coincidence formula in the non-oriented case). For any pair of maps between closed manifolds $f, g: M \rightarrow N$ with $g$ orientation true,

$$
L(f, g)=\operatorname{ind}(f, g) \text {. }
$$

7. Defective classes and obstruction theory. The problem "deform the pair $f, g$ : $M \rightarrow N$ to a coincidence free pair" is equivalent to "deform the map $M \ni x \rightarrow(f x, g x) \in$ $N \times N$ outside the diagonal $\Delta N \subset N \times N$ ". To the last question one may apply the obstruction theory. E. Fadell and S. Husseini presented such an approach for fixed points $(f=i d)$ in $[\mathrm{FH}]$. Then R. Dobreńko generalized it to coincidences in the oriented case [D]. The obstruction which he obtained can after some natural isomorphisms be interpreted as the formal sum

$$
\sum_{A} \operatorname{ind}(f, g ; A) \cdot A \in \oplus_{A} \mathbf{Z}
$$

where the summation is over the set of Reidemeister classes of the pair $f, g[\mathrm{D}]$. Recently D. L. Gonçalves described this obstruction in the general case [G]. Here we present the main points of this description since it also reflects the role of defective classes. 
The obstruction to deforming the above map $(f, g)$ into $N \times N-\Delta N$ yields an obstruction $o(f, g) \in H^{n}(M ; \mathbf{Z}[\pi])$ where $\mathbf{Z}[\pi]$ denotes the local system in which $\pi_{1} M$ acts on $\pi=\pi_{1} N$ by the formula

$$
\alpha \circ \theta=\operatorname{sgn}\left(g_{\#}(\alpha)\right) \cdot f_{\#}(\alpha) \cdot \theta \cdot g_{\#}(\alpha)^{-1},
$$

$\alpha \in \pi_{1} M, \theta \in \pi_{1} N[\mathrm{FH}],[\mathrm{D}],[\mathrm{G}]$. Then

$$
H^{n}(M ; \mathbf{Z}[\pi]) \approx \oplus \mathbf{Z} \oplus \mathbf{Z}_{2}
$$

where the components are indexed by Reidemeister classes: non-defective classes correspond to $\mathbf{Z}$-summands while defective classes correspond to $\mathbf{Z}_{2}$-summands. Moreover under this isomorphism

$$
o(f, g)=\sum_{A} k_{A} \cdot A \in \oplus \mathbf{Z} \oplus \mathbf{Z}_{2}
$$

where $k_{A}= \pm|i n d|(f, g: A)$. More exactly if $M, N$ are orientable then there is a natural isomorphism under which $k_{A}=\operatorname{ind}(f, g ; A)$ and we get the formula from [D]. Such a formula also holds if we assume that one of the maps is orientation true. In the general case however there is no natural isomorphism and the sign of $k_{A}$ depends on the choice of the isomorphism $H^{n}(M ; \mathbf{Z}[\pi]) \approx \oplus \mathbf{Z} \oplus \mathbf{Z}_{2}$.

8. Fibre maps. Computations of the Nielsen fixed point number turned out to be hard in general. In 1967 R. Brown considered a fibre map of a locally trivial fibre bundle and asked about a relation among the Nielsen numbers of: the total map, its restriction to the fibre and the map of base spaces ("product formula") [B]. This problem was also treated in numerous papers in the 70s and finally Chen Ye You found a necessary and sufficient condition for this formula $[Y]$. This result gave later formulae for the Nielsen number of self-maps of tori, nilmanifolds, certain solvmanifolds and some other $K(\pi, 1)$ spaces.

It turns out that the result of You can be modified to coincidences in the oriented case [Je1] but its natural extension without the orientability assumption is not valid [Je2]. We will not formulate here the corresponding conditions but we will only discuss the so called "index product formula" (8.1). This formula holds in the oriented case (we sketch the proof) but it fails in non-oriented case (we give a counter-example). In fact it turns out that this index product formula (and as a consequence the "product formula" for the Nielsen numbers) holds if there is no defective class [Je2].

First a few words on the "Nielsen number product formula". Let $p: E \rightarrow B, p^{\prime}$ : $E^{\prime} \rightarrow B^{\prime}$ be fibre bundles whose base spaces and fibres are closed oriented manifolds of respectively equal dimensions. Consider a pair of fibre maps, i.e. a commutative diagram

$$
\begin{array}{ccc}
E & \stackrel{f, g}{\longrightarrow} & E^{\prime} \\
p \downarrow & & p^{\prime} \downarrow \\
B & \stackrel{\bar{f}, \bar{g}}{\longrightarrow} & B^{\prime}
\end{array}
$$

Choose a coincidence point from each essential class of $C(\bar{f}, \bar{g})$ :

$$
x_{i} \in A_{i} \subset C(\bar{f}, \bar{g}), \quad i=1, \ldots, k=N(\bar{f}, \bar{g}) .
$$


Then the "product formula" takes the form

$$
N(f, g)=N\left(f_{1}, g_{1}\right)+\ldots+N\left(f_{k}, g_{k}\right)
$$

where $f_{i}, g_{i}$ denote the restrictions of $f, g$ to the fibres $E_{b_{i}} \rightarrow E_{f b_{i}}^{\prime}$ where $E_{b}=p^{-1}(b)$.

Now we prove the index product formula in the oriented case (Thm 5.5 in [Je1]).

THEOREM 8.1. Consider the above diagram where all the spaces involved are closed oriented manifolds of respectively the same dimensions. Let $A \subset E$ be a Nielsen class of $f, g$, let $\bar{A} \subset B$ be a Nielsen class containing $p A$ and let $b \in p \bar{A}$. Then

$$
\operatorname{ind}(f, g ; A)=\operatorname{ind}(\bar{f}, \bar{g} ; \bar{A}) \cdot \operatorname{ind}\left(f_{b}, g_{b} ; A \cap B_{b}\right) \text {. }
$$

SketCH of PROOF. Since index is a homotopy invariant, we may assume that $C(f, g)$ is finite. By the Homotopy Covering Property we may assume that $C\left(f_{b}, g_{b}\right)$ is finite for any $b \in C(\bar{f}, \bar{g})$. Moreover by the local triviality we may assume that for any $x \in C(f, g)$ there exist euclidean neighbourhoods $U, V, \bar{U}, \bar{V}$ satisfying $x \in U \subset E, \quad f x=g x \in$ $V \subset E^{\prime}, \quad \bar{U}=p U \subset B, \quad \bar{V}=p V \subset B^{\prime}, \quad U \approx \bar{U} \times R^{n}, \quad V \approx \bar{V} \times R^{n}$ and $f(x)=$ $(\bar{f}(\bar{x}), \phi(t)), \quad g(x)=(\bar{g}(\bar{x}), \psi(t)))$ where $x=(\bar{x}, t) \in \bar{U} \times R^{n} \approx U$. Now

$$
\operatorname{ind}(f, g ; x)=\operatorname{ind}(\bar{f}, \bar{g} ; \bar{x}) \operatorname{ind}(\phi, \psi ; t) .
$$

On the other hand we notice that $N\left(f_{b}, g_{b} ; E_{b} \cap A\right)$ are equal for all $b$ lying in the same Nielsen class of $\bar{f}, \bar{g}$. Now by additivity of the index

$$
\begin{aligned}
\operatorname{ind}(f, g ; A) & =\sum_{x \in A} \operatorname{ind}(f, g ; x)=\sum_{\bar{x} \in \bar{A}}\left(\sum_{x \in E_{\bar{x}} \cap A} \operatorname{ind}(f, g ; x)\right) \\
& =\sum_{\bar{x} \in \bar{A}}\left(\sum_{(\bar{x}, t) \in E_{\bar{x}} \cap A} \operatorname{ind}(\bar{f}, \bar{g} ; \bar{x})\right) \cdot \operatorname{ind}\left(f_{\bar{x}}, g_{\bar{x}} ; t\right) \\
& =\left(\sum_{\bar{x} \in \bar{A}} \operatorname{ind}(\bar{f}, \bar{g} ; \bar{x})\right) \cdot\left(\sum_{(\bar{x}, t) \in E_{\bar{x}} \cap A} \operatorname{ind}\left(f_{\bar{x}}, g_{\bar{x}} ; t\right)\right) \\
& =\left(\sum_{\bar{x} \in \bar{A}} \operatorname{ind}(\bar{f}, \bar{g} ; \bar{x})\right) \cdot \operatorname{ind}\left(f_{b}, g_{b} ; A \cap E_{b}\right)=\operatorname{ind}(\bar{f}, \bar{g} ; \bar{A}) \cdot \operatorname{ind}\left(f_{b}, g_{b} ; A \cap E_{b}\right)
\end{aligned}
$$

for a fixed $b \in \bar{A}$.

Notice that the above proof is not valid for the semi-index because then the additivity property does not hold. The following example shows that this formula is not true in general.

EXAMPLE 8.2. Let $M$ be a non-orientable two-dimensional connected 2-manifold. It may be regarded as a CW-complex with a unique 2-cell. Let $f^{\prime}: M \rightarrow S^{2}$ be a map sending the 1-skeleton to a point $y_{1} \in S^{2}$ and the interior of the 2-cell homeomorphically onto $S^{2}-y_{1}$. Let $g^{\prime}: M \rightarrow S^{2}$ denote the constant map with $g^{\prime}(M)=y_{0} \neq y_{1}$. Then the pair $\left(f^{\prime}, g^{\prime}\right)$ is transverse and $C(f, g)$ consists of a single point $x_{0}$. This point is selfreducing hence it forms a defective class (compare Ex. 4.1). On the other hand consider the maps $\bar{f}, \bar{g}: S^{2} \rightarrow S^{2}$ where $\bar{f}=i d_{S^{2}}, \bar{g}(x, y, z)=(-x,-y, z)$. This pair is also transverse and $(\bar{f}, \bar{g})=\{(0,0,1),(0,0,-1)\}=\left\{b_{0}, b_{1}\right\}$ are two Nielsen equivalent points which are not R-related (since $S^{2}$ is orientable). Notice that $\mid$ ind $\mid\left(f^{\prime}, g^{\prime}\right)=1, N\left(f^{\prime}, g^{\prime}\right)=1$ 
and $\mid$ ind $\mid(\bar{f}, \bar{g})=2, N(\bar{f}, \bar{g})=1$. Consider the diagram

$$
\begin{array}{ccc}
S^{2} \times M & \stackrel{f, g}{\longrightarrow} & S^{2} \times S^{2} \\
p_{1} \downarrow & & \downarrow p_{1} \\
S^{2} & \stackrel{\bar{f}, \bar{g}}{\longrightarrow} & S^{2}
\end{array}
$$

where $f=\bar{f} \times f^{\prime}, g=\bar{g} \times g^{\prime}$ and $p_{1}$ denotes the projection on the first factor. Then $C\left(\bar{f} \times f^{\prime}, \bar{g} \times g^{\prime}\right)=\left\{\left(b_{0}, x_{0}\right),\left(b_{1}, x_{1}\right)\right\}$ and the two points are Nielsen equivalent. Each of them is self-reducing (since so is $x_{0} \in C\left(f^{\prime}, g^{\prime}\right)$ ) hence $\mid$ ind $\mid(f, g)=0 \neq 2 \cdot 1=$ $\mid$ ind $|(\bar{f}, \bar{g}) \cdot|$ ind $\left|\left(f^{\prime}, g^{\prime}\right)\right|$.

It turns out that the defective classes are the unique obstruction: theorem (3.13) in [Je2] states that if neither $\bar{A}$ nor any class in $E \cap A$ is defective then the analogous semi-index product formula holds. This gives rise to a generalization of the "product formula" to the non-oriented case [Je2;4.3].

9. Nielsen number and covering spaces. Another method of computing Nielsen number is lifting the given maps to covering spaces and finding relations between their Nielsen numbers. More precisely we consider a commutative diagram

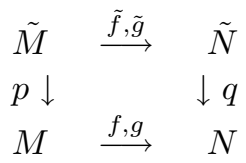

where all the spaces involved are closed $n$-manifolds and $p, q$ are regular coverings. A relation between $N(f, g)$ and the Nielsen numbers of lifts is described in [Je3] (Thm. 2.5). Here we will sketch a very special case of this theorem (Corollary (2.6) in [Je3]).

THEOREM 9.1. If $\pi_{1} N=\mathbf{Z}_{2}, \tilde{N}$ is the two-fold connected cover and no Nielsen class of $f, g$ is defective then

$$
N(f, g)= \begin{cases}N(\tilde{f}, \tilde{g}) & \text { if } f_{\#} \neq g_{\#}, \\ N(\tilde{f}, \tilde{g})+N(\tilde{f}, \beta \tilde{g}) & \text { if } f_{\#}=g_{\#},\end{cases}
$$

where $\beta$ is the involution of the two-fold cover $\tilde{N}$.

Proof. We may assume that the pair $f, g$ is transverse. Then any pair of lifts is also transverse. Suppose that a path $\omega$ establishes the Nielsen relation between two coincidence points $x_{0}, x_{1} \in C(f, g)$ and assume that $p^{-1}\left(x_{0}\right) \cap C(\tilde{f}, \tilde{g})=\left\{\tilde{x}_{01}, \ldots, \tilde{x}_{0 k}\right\}$ is not empty. Let $\bar{\omega}_{i}$ be the lift of $\omega$ starting from $\tilde{x}_{0 i}$. Then $p^{-1}\left(x_{1}\right) \cap C(\tilde{f}, \tilde{g})=\left\{\tilde{\omega}_{1}(1), \ldots, \tilde{\omega}_{k}(1)\right\}$. If moreover $\omega$ establishes the R-relation between $x_{0}$ and $x_{1}$ then $\tilde{\omega}_{i}$ establishes the Rrelation between $\tilde{\omega}_{i}(0)$ and $\tilde{\omega}_{i}(1)(i=1, \ldots, k)$ (since $p, q$ are local homeomorphisms). This implies that if a Nielsen class $A \subset C(f, g)$ is not essential then

$$
|i n d|\left(\tilde{f}, \tilde{g} ;\left(p^{-1} A\right) \cap C(\tilde{f}, \tilde{g})\right)=0 .
$$

Now assume that $A$ is essential. By the above we may assume that $A=\left\{x_{0}, \ldots, x_{l}\right\}$ and no two points are R-related. Suppose that $p^{-1}\left(x_{0}\right) \cap C(\tilde{f}, \tilde{g})=\left\{\tilde{x}_{01}, \ldots, \tilde{x}_{0 k}\right\}$ is not empty. As above we may denote $p^{-1}\left(x_{i}\right) \cap C(\tilde{f}, \tilde{g})=\left\{\tilde{x}_{i 1}, \ldots, \tilde{x}_{i k}\right\}$ and we notice that no $\tilde{x}_{i s}, \tilde{x}_{j t}$ are R-related, since otherwise this would imply $x_{i} R x_{j}$ (to get this contradiction 
for $i=j$ we need the assumption that $x_{i}$ is not self-reducing). Thus for any essential class $A \subset C(f, g)$

$$
\mid \text { ind } \mid\left(\tilde{f}, \tilde{g} ;\left(p^{-1} A\right) \cap C(\tilde{f}, \tilde{g})\right)=0 \quad \text { iff } \quad p^{-1} A \cap C(\bar{f}, \bar{g})=\emptyset .
$$

On the other hand notice that $\pi_{1} \tilde{N}=0$ hence $C(\tilde{f}, \tilde{g})$ is one Nielsen class (possibly empty), which implies that $\left(p^{-1} A\right) \cap C(\tilde{f}, \tilde{g})=C(\tilde{f}, \tilde{g})$ or is empty. Combining the above two results we get

$$
N(\tilde{f}, \tilde{g})=1 \quad \text { iff } \quad|i n d|(\tilde{f}, \tilde{g}) \neq 0 \quad \text { iff } \quad p(C(\tilde{f}, \tilde{g})) \subset C(f, g) \text { is essential. }
$$

It remains to notice that:

- If $f_{\#} \neq g_{\#}$ then there is only one Nielsen class $C(f, g)=p(C(\tilde{f}, \tilde{g}))$.

- If $f_{\#}=g_{\#}$ then there are two Nielsen classes $A_{0}=p(C(\tilde{f}, \tilde{g})), A_{1}=p(C(\tilde{f}, \beta \tilde{g}))$.

The above arguments are not valid for defective classes. However in some particular cases the defective classes can be effectively detected by the use of the modulo 2 Lefschetz number. For example if $C(f, g)$ is exactly one Nielsen class and is defective then $\mid$ ind $\mid(f, g)=L_{2}(f, g)$. This observation and Theorem 2.9 allow one to derive formulae for the coincidence Nielsen number for maps into the real-projective spaces [Je3].

\section{References}

[B] R. F. Brown, The Lefschetz Fixed Point Theorem, Glenview, New York, 1971.

[D] R. Dobreńko, The obstruction to the deformation of a map out of a space, Dissertationes Math. (Rozprawy Mat.) 295 (1990).

[DJ] R. Dobreńko and J. JezIERSKI, The coincidence Nielsen theory on non-orientable manifolds, Rocky Mountain J. Math. 23 (1993), 67-85.

[DK] R. Dobreńko and Z. Kucharski, On the generalization of the Nielsen number, Fund. Math. 134 (1990), 1-14.

[Dl] A. Dold, Lectures on Algebraic Topology, Springer, New York, 1972.

[G] D. L. GonçAlves, Indices for coincidence classes and the Lefschetz formula for nonoriented manifolds, preprint, Math. Institut, Univ. Heidelberg.

[GJ] D. L. GonÇALves and J. JEzIERSKI, Lefschetz coincidence formula on non-orientable manifolds, Fund. Math. 153 (1997), 1-23.

[H] M. HiRsch, Differential Topology, Springer, New York, 1976.

[Je1] J. JeZIERsKI, The Nielsen number product formula for coincidences, Fund. Math. 134 (1989), 183-212.

[Je2] J. Jezienski, The semi-index product formula, Fund. Math. 140 (1992), 99-120.

[Je3] J. JezIERSKI, The coincidence Nielsen number for maps into real projective spaces, Fund. Math. 140 (1992), 121-136.

[Je4] J. JezIERski, The Nielsen coincidence theory on topological manifolds, Fund. Math. 143 (1993), 167-178.

[Ji1] B. J. JiAng, Lectures on the Nielsen Fixed Point Theory, Contemp. Math. 14, Amer. Math. Soc., Providence, 1983.

[Ji2] B. J. JiAnG, Fixed point classes from a differential viewpoint, in: Lecture Notes in Math. 886, Springer, 1981, 163-170. 
[L] S. LEFSCHETZ, Intersections and transformations of complexes and manifolds, TAMS 28 (1926) 1-49.

[N1] J. NiELSEN, Über die Minimalzahl der Fixpunkte bei Abbildungstypen der Ringflächen, Math. Ann. 82 (1929), 83-93.

[N2] J. NiELSEN, Untersuchungen zur Topologie der geschlossenen zweiseitigen Flächen, I, II, III, Acta Math. 50 (1927), 189-358; 53 (1929), 1-76; 58 (1932), 87-167.

[Sch] H. Schirmer, Mindestzahlen von Koinzidenzpunkten, J. Reine Angew. Math. 194 (1955), 21-39.

[Sp1] E. Spanier, Algebraic Topology, McGraw-Hill, New York, 1966.

[Sp2] E. Spanier, Duality in topological manifolds, in: Colloque de Topologie Tenu à Bruxelles (Centre de Recherche Mathématiques), 1966, 91-111.

[V] J. Vick, Homology Theory, Academic Press, New York, 1973.

[Y] C. Y. You, Fixed points of a fibre map, Pacific J. Math. 100 (1982), 217-241. 